\title{
The association between female reproductive tract microbiota and recurrent pregnancy loss: a nested case-control study
}

Pirkko Peuranpää ( $\nabla$ pirkko-liisa.peuranpaa@helsinki.fi )

The Department of Obstetrics and Gynecology, University of Helsinki and Hyvinkää Hospital, Hyvinkää, Finland https://orcid.org/0000-0002-6841-0757

\section{Research Article}

Keywords: Recurrent pregnancy loss, recurrent miscarriage, microbiota, endometrium, vagina

Posted Date: December 14th, 2021

DOI: https://doi.org/10.21203/rs.3.rs-1167008/v1

License: (c) (i) This work is licensed under a Creative Commons Attribution 4.0 International License.

Read Full License 


\section{Abstract \\ Objective}

To study the associations of endometrial and vaginal microbiota with recurrent pregnancy loss (RPL).

\section{Design}

A nested case-control study.

\section{Setting}

Helsinki University Hospital, Finland.

\section{Population}

Women with two or more consecutive pregnancy losses $(n=47)$ and healthy control women without a history of pregnancy loss $(n=39)$.

\section{Methods}

The endometrial and vaginal microbiota compositions, analysed using 16S rRNA gene amplicon sequencing, were compared between RPL women and controls, and between individual vaginal and endometrial samples. The mycobiota composition was analysed using internal transcribed spacer 1 amplicon sequencing for a descriptive summary. The models were adjusted for confounding variables $\mathrm{BMI}$, age, and parity, and FDR-corrected $P$-values ( $q$-values) were used to define nominal statistical significance at $q<0.05$.

\section{Main outcome measures}

Mean relative microbial abundances.

\section{Results}

Lactobacillus crispatus was less abundant in the RPL endometrial samples compared to controls (mean relative abundance $17.2 \%$ vs. $45.6 \%, q=0.04$ ). Gardnerella vaginalis was more abundant in RPL women than in controls in both endometrial $(12.4 \%$ vs. $5.8 \%, q<0.001)$ and vaginal samples $(8.7 \%$ vs. $5.7 \%, q<$ 
0.01). The individual vaginal and endometrial microbial composition correlated strongly $(\mathrm{R}=0.85, P<$ 0.001). Fungi were detected in $22 \%$ of the endometrial and $36 \%$ of the vaginal samples.

\section{Conclusions}

Unfavourable reproductive tract microbiota was associated with RPL and may represent a novel risk factor for pregnancy losses.

\section{Introduction}

Recurrent pregnancy loss (RPL) is one of the most challenging issues in reproductive medicine because its causes are often unknown, and effective treatment is rarely available. European Society of Human Reproduction and Embryology (ESHRE) defines RPL as the spontaneous loss of two or more pregnancies (1), and it affects $1-3 \%$ of couples trying to have a child. Recognised causes for RPL are chromosomal abnormalities, uterine malformations, antiphospholipid syndrome, and endocrinological disorders (2), while over half of the RPLs still remain unexplained.

Several studies have suggested an infectious etiology behind miscarriages $(3,4)$. Chronic endometritis has been associated with RPL (5), and bacterial vaginosis (BV) has been linked to the risk of miscarriage $(6,7)$. Recent studies using high-throughput DNA sequencing techniques have shown that Lactobacillus spp. dominate the vaginal bacterial composition in healthy early pregnancy $(8,9)$, while the reduced prevalence of lactobacilli has been associated with pregnancy loss (10). Mycobiota, consisting of various species of fungi, is another important element of the vaginal ecosystem (11). However, there are no studies examining mycobiota in relation to reproductive outcomes.

The uterine cavity has long been thought to be sterile, but recent studies have reported that endometrium may have a distinct microbiome $(12,13)$. Like in the vagina, the dominant species in the endometrium are usually lactobacilli (12), and alteration of this composition may affect reproductive outcomes. In patients treated with in vitro fertilization (IVF), non-Lactobacillus dominated endometrial microbiota has been associated with lower implantation rates, clinical pregnancy rates, and live birth rates compared to Lactobacillus-dominated microbiota (14). The impact of endometrial microbiota on RPL has not been investigated before.

The goal of our study was to explore the composition of microbiota and mycobiota in endometrial and vaginal samples in women with RPL and compare the results to healthy women without a history of miscarriages. We also investigated whether the composition of vaginal microbiota reflects the composition of endometrial microbiota.

\section{Material And Methods}

\section{Study population}


TOIVE is a prospective cohort study of the immunological and microbiological causes of RPL, conducted at the University of Helsinki and Helsinki University Hospital (HUS), Finland. Between March 2018 and June 2020, we recruited 51 women referred to the Reproductive Medicine Unit and the Department of Obstetrics and Gynecology of Hyvinkää Hospital due to RPL (Figure 1). Women were eligible if they had a history of three or more consecutive clinical first-trimester pregnancy losses or two losses, with at least one of them in the second trimester. We defined clinical pregnancy loss as a spontaneous loss of an intrauterine pregnancy confirmed by an ultrasound, or positive urine or serum human chorionic gonadotrophin and over six weeks of amenorrhea.

As controls, we recruited 40 women investigated for male factor infertility between June 2018 and December 2020. They represented healthy Finnish women since they had no history of pregnancy losses, endometriosis, anovulation, or fallopian tube defects. Participants were excluded if they were $<18$ or $\geq 40$ years of age, had hepatitis or HIV infection, or had an irregular menstrual period ( $<21$ or $>42$ days).

Power calculations were not applicable to our associative and novel setting since earlier studies on microbiota composition and RPL are lacking.

\section{Collection of clinical data}

All participants gave their written informed consent. Upon enrolment, we took a detailed medical and reproductive history, and participants received a questionnaire related to previous infections, the use of anti- or probiotics, sexual behaviour, and educational background. The basic examinations for RPL included transvaginal 2D-ultrasound, complete blood count, phospholipid antibodies, karyotyping (of both partners), thyroid function tests, thyroid peroxidase antibodies, fasting glucose, and glycosylated haemoglobin. Screening for thrombophilia, celiac disease, hyperprolactinemia, or uterine anomalies with 3D-ultrasound or hysteroscopy were performed when necessary. Women were not routinely tested for sexually transmitted diseases nor chronic endometritis.

\section{Endometrial and vaginal sampling}

We collected vaginal and endometrial samples in the mid-luteal phase, 6-8 days after a positive ovulation test (Clearblue Digital ${ }^{\circledR}$, Swiss Precision Diagnostics $\mathrm{GmbH}$, Switzerland). Couples were advised to use a condom or abstain from sexual intercourse during the menstrual cycle in which the samples were taken for contraception and to avoid the effects of seminal fluid on the microbiota. Vaginal samples were collected in a speculum examination from the right and left fornix with sterile flocked swabs (FLOQSwabs, Copan spa ${ }^{\circledR}$, Italy) and severed to $1.5 \mathrm{~mL}$ Eppendorf tubes. We used non-sterile examination gloves and a white coat during sampling, but not a mask or hair cover. Lubricants were not used. Endometrial samples were collected to $1.5 \mathrm{~mL}$ Eppendorf tubes with endometrial biopsy curette (Pipelle ${ }^{\circledR}$, Prodimed, France) without contact to vaginal walls. Eppendorf tubes were frozen at $-20^{\circ} \mathrm{C}$ immediately after sampling and moved to $-80^{\circ} \mathrm{C}$ within two weeks.

\section{DNA extraction}


Microbial DNA was extracted from the vaginal samples using a beat beating method as previously described (15). One-sixth of the endometrium biopsies (average weight $33 \mathrm{mg}$; SD 22) was used for DNA extraction with negative controls using the same method as for the vaginal samples. DNA was quantified using Quanti-iT Pico Green dsDNA Assay (Invitrogen ${ }^{\circledR}$, San Diego, CA, USA). The DNA yields for both sample types were comparable; mean 78.8 (SD 60) $\mathrm{ng} / \mu \mathrm{l}$ in vaginal and 43.8 (SD 30) $\mathrm{ng} / \mu \mathrm{l}$ in endometrial samples. The negative controls (no input sample for DNA extraction) did not contain detectable amounts of DNA.

\section{S rRNA gene and Internal transcribed spacer (ITS) amplicon sequencing}

Illumina MiSeq paired-end sequencing of PCR-amplicons from the hypervariable V3-V4 regions of the bacterial 16S rRNA gene (primers 341F/785R) and fungal ITS-1 region (primers ITS1F and ITS2) was prepared and performed as explained in detail elsewhere (16). Briefly, bacterial and fungal amplicons were prepared separately, combined for indexing in a 1:1 ratio, and an equimolar pool was sequenced using $2 \times 300$ bp reads and a MiSeq v3 reagent kit at the Biomedicum Functional Genomics Unit (FuGU), Helsinki, Finland.

\section{Sequence processing and analysing}

The primary step of the analysis was to split the combined 16S rRNA gene and ITS sequence fastq-files into separate datasets. This was done by first removing ambiguous $(\mathrm{N})$ bases from the reads, followed by primer-based separation using cutadapt v3.5, removing the primers in the process. From this step onwards, all pre-processing was carried out individually on both datasets. The bacterial 16S rRNA gene dataset was processed using dada2 v1.20, following the pipeline tutorial v1.16, while the ITS pipeline tutorial v1.8 was used for processing the ITS sequence dataset.

The dada2 tutorials were only followed until ASV table construction and removal of chimeras, after which the taxminer package was used to assign taxonomic annotations (17). This is a BLAST-based annotation tool combined with text-mining-based filtration to assign the most likely annotations. A detailed description of this approach has been communicated elsewhere (16).

\section{Statistical analysis}

Statistical analyses were performed using IBM SPSS Statistics, version 25 (IBM Corporation, Armonk, NY, USA) software for univariate data. Two-sample t-test and Mann-Whitney $U$ test compared the continuous background variables, Pearson chi-square, and Fisher's exact tests compared the categorical variables between RPL women and controls. Parity was categorized as nulliparous and parous ( $\geq 1$ delivery).

The primary outcomes were mean relative abundances of bacteria and fungi in the endometrial and vaginal samples. Associations between background variables and the microbiota were analysed with permutational analysis of variance (PERMANOVA) for the overall microbiota variation using the adonis2 function with 99999 permutations from the vegan package (18). Potential confounders to adjust models 
for differential abundance analysis downstream were identified based on the following criteria: 1) factors previously identified to influence microbiota and RPL and 2) confounders with statistical significance, determined by PERMANOVA analysis, ordination plots, and the GroupTest function from the package mare (19). GroupTest performs differential abundance analysis for taxa within user-defined groups, in this case each background variable. The function finds the most suitable model for individual taxa and uses read counts as an offset within the model formula to account for sequencing depth. Lastly, cases and controls were used as the grouping variable in GroupTest to determine the significantly different taxa in endometrial and vaginal samples between RPL and control women. Based on the criteria mentioned above, body mass index (BMI), parity, and age were selected as confounders. The $P$-values obtained were adjusted for multiple testing by False Discovery Rate (FDR)-correction and reported as $q$-values. The relationship between individual vaginal and endometrial microbiota was investigated using Pearson correlation coefficient.

\section{Results}

We sampled 47 women with RPL and 39 control women to study the composition of the endometrial and vaginal microbiota and mycobiota. In one RPL woman, only the vaginal sample was obtained. RPL women had a history of three (range 2-5) consecutive miscarriages, of which 13 (15.3\%) had occurred during the second trimester of pregnancy. Nine women had a likely explanation for their miscarriages: five were diagnosed with congenital uterine malformation, three with acquired thrombophilia, and one with antiphospholipid syndrome and chromosomal translocation.

The RPL women were older (mean 33.2 [22 - 39] vs. 32.1 [26 - 38] years, $P=0.04$ ), had a higher BMI (mean $25.4[19.5-39.4]$ vs. $23.3[19.4-33.1] \mathrm{kg} / \mathrm{m} 2, P=0.02$ ), and were parous more often than the controls $(50.0 \%$ vs. $15.4 \%, P<0.001)$. The RPL women had more often self-reported recurrent $(\geq 3)$ candida vaginitis $(19.0 \%$ vs. $0 \%, P=0.006), \mathrm{BV}(16.7 \%$ vs. $0 \%, P=0.01)$, and regular oral or vaginal probiotics use $(36.6 \%$ vs. $10.5 \%, P=0.008)$ than the controls. The clinical characteristics of the study population are shown in Table S1.

\section{S rRNA gene sequencing results}

After taxonomic annotations and quality filtration (>500 reads), 74\% (34/46) of the RPL and 77\% (30/39) of control women's endometrial samples remained for bacterial analysis with an average read count of 4 500 (535-20 829), and 4500 (569-11 540), respectively. Similarly, 98\% of the vaginal RPL samples (46/47) and $100 \%$ of controls (39/39) remained, with an average read count of 23600 (3 728-47 923) and 24300 (1 322-55 011).

PERMANOVA analysis showed a difference in the overall endometrial microbiota composition between the RPL and control women ( $R 2=0.050, P=0.01$, Figure $S 1$, Table S2), but the vaginal bacterial compositions were similar between the groups (Figure S2, Table S2). BMI was a strong clinical explanatory factor for the endometrial microbiota $(R 2=0.057, P=0.005)$, especially among RPL women 
( $R 2=0.09, P=0.005)$. Current vaginitis symptoms, use of vitamin $D$ and folic acid, microbiota diversity, and read count explained the variability of the vaginal microbiota in the whole study population, and among the RPL women also BMI, age, and probiotics. Parity, gravidity, or curettages had no impact on the endometrial or vaginal microbiota variation.

In the endometrial samples, 37 bacterial species were identified (Figure 2A). L. crispatus was significantly less and $L$. jensenii more abundant in the RPL women compared to controls (mean relative abundance of L. crispatus $17.2 \%$ vs. $45.6 \%$, respectively, $q=0.04$, L. jensenii $5.6 \%$ vs. $3.6 \%, q<0.01$ ) (Figure 3, Table S3). L. iners was the most dominant endometrial bacterium in the RPL women (mean relative abundance $32.2 \%$ vs. $20.0 \%, q>0.05$ ). Gardnerella vaginalis was more abundant in the RPL women compared to controls $(12.4 \%$ vs. $5.8 \%, q<0.001)$. None of the intestinal bacteria, such as Escherichia coli, Blautia spp., and Faecalibacteria spp., nor the uncultured bacteria (bacteria that lack culture-based genomic and physiological characterization) were significantly differentially abundant in the RPL women compared to the controls.

In the vaginal samples (Figure 2B), the RPL women had more G. vaginalis (mean relative abundance $8.7 \%$ in RPL women vs. $5.7 \%$ in controls, $q<0.01$ ) and less $G$. Leopoldii (1.0\% vs. $3.2 \%$, respectively, $q<0.001$ ), whereas L. crispatus was the most abundant bacterium (35.1\% vs. $47.5 \%, q>0.05$ ) (Figure 3, Table S3). In the pooled endometrial and vaginal samples, $L$. crispatus was less and $G$. vaginalis more abundant in RPL women than in controls (L. crispatus $27.5 \%$ vs. $46.6 \%, q=0.04$; G. vaginalis $10.2 \%$ vs. $5.7 \%, q<$ $0.001)$.

There were three blank samples within this pipeline that were analysed with the same pre-processing and filtration criteria. Only two out of these three samples had a considerable number of reads, which totalled to $\sim 8500$ (Figure S3). These annotated to Streptococcus oralis ( 4000), Fannyhessia vaginae ( 600), Prevotella amnii ( 1700), and Sneathia vaginalis ( 1300). S. oralis, a potential oral contaminant, was not detected in any other samples. F. vaginae, $P$. amnii, and $S$. vaginae are known vaginal microbes, and their presence in the blank sample is most likely due to contamination from the primary study samples. Due to these reasons, we did not subtract these reads from the sample data. Furthermore, none of these bacteria were significant in our downstream analysis, and therefore not crucial for the main study comparisons.

\section{ITS sequencing results}

After taxonomic annotations and quality filtration, 24\% (11/46) of RPL and 21\% (8/39) of control women's endometrial samples remained for the fungal analysis, with an average read count of $\sim 14500$ (1031-81 809) and 12 000 (1383-23 999) respectively. Similarly, 36\% (17/47) of the RPL and 36\% $(14 / 39)$ of control women's vaginal samples remained after filtration, with an average read count of $\sim 6000$ (742-22 500) and 13000 (542-30 685). Since fungi could only be detected in a small fraction of the samples, they may not sufficiently represent the entire study cohort. We therefore refrain from statistical analysis and present a descriptive overview of the taxonomic profiles. 
The most prevalent genus was Candida, which was detected in 19/39 samples (Figure S4). C. albicans was not detected in RPL women's endometrium, although it was the most abundant taxa in their vaginal samples (mean relative abundance $26.9 \%$ ) and in control women's endometrial and vaginal samples (mean relative abundance $49.9 \%$ and $42.9 \%$, respectively). On the other hand, C. parapsilosis was only detected in RPL women, with an average relative abundance of $18.2 \%$ in endometrial and $11.8 \%$ in vaginal samples.

\section{Comparison of microbiota in vaginal and endometrial samples}

To study the relationship between microbiota colonizing endometrium and vagina, we compared samples collected from these anatomical sites of the same woman. We found a strong within-individual correlation between vaginal and endometrial microbiota composition (mean Pearson's correlation coefficient 0.85, $P<0.001$, Figure 4, Figure S5). The overlap was strongest in Lactobacillus-dominated samples, while intestinal and uncultured bacteria were more abundant in the endometrium, especially in RPL women.

\section{Discussion}

\section{Main Findings}

We observed an association between RPL, reduced L. crispatus and increased G. vaginalis abundance in the endometrium, and an increased $G$. vaginalis abundance in the vagina. The composition of vaginal microbiota was in concordance with the endometrial microbiota.

\section{Strengths and limitations}

To our knowledge, this is the first study comparing RPL women's endometrial microbiota with healthy controls. Our study population was ethnically and even genetically homogenous. Sampling was timed to the mid-secretory phase to analyse the microbiota during the receptive state of the endometrium, since the composition of vaginal $(20)$ and endometrial microbiota $(21,22)$ may vary throughout the menstrual cycle. We used a recommended technique, transcervical biopsy, for collecting endometrial tissue (23) because biopsies represent mucosal microbiota better than superficial samples, such as swabs or endometrial fluid (24). In addition to the microbiota, we also analysed the endometrial mycobiota, which has not been previously explored. Our control women represent the general population as closely as possible since they were healthy and did not have previous miscarriages or conditions known to associate with alterations in reproductive tract microbiota, such as endometriosis $(21,25)$, endometrial polyps (26), PCOS (27), or fallopian tube occlusion (7). We also studied the influence of various lifestyle factors on our findings. However, the applicability of our results to the general population remains to be explored. 
As a limitation, low microbial abundance specimens, such as the endometrium, are susceptible to contamination (28). Although we avoided contact with the vagina during sampling, cervicovaginal contamination cannot be ruled out since similarities existed between individual vaginal and endometrial samples. However, growing evidence supports the theory that vaginal and endometrial ecosystems are not separate, but can share microbes $(12,29,30)$, and only a minority of studies have questioned the existence of a uterine microbiota (31). Although cervical mucus protects the uterine environment, sperm pass from the vagina to the uterus and vaginally administered radioactively labelled albumin macrospheres spread in the uterine cavity within minutes (32). Therefore, it is likely that microbes ascending from the vagina may colonize the endometrium. Also, the composition of endometrial microbiota has been reported to be highly similar in laparoscopically and trans-cervically taken samples (12), and common vaginal microbes, including lactobacilli, have been found in the endometrium even after hysterectomy $(12,33,34)$. Overall, even if there were cervicovaginal contamination of our endometrial samples, it does not explain the observed differences between RPL women and controls.

Environmental contamination was not likely since bacteria found in the negative controls, such as $S$. oralis, were not found in women's samples, while reads for Lactobacillus and Gardnerella species were not substantial. The presence of vaginal microbes such as $F$. vaginae, $P$. amnii, and $S$. vaginae could be explained as contamination, however, considering their low abundance and prevalence in the study samples, as well as lack of significance within the group comparisons indicates that they had not significant effect on the overall results.

\section{Interpretation}

Our finding of a reduced abundance of L. crispatus in RPL endometrial samples is in line with prior studies reporting that in vagina, $L$. crispatus dominance is considered a healthy microbial environment $(35,36)$. Furthermore, L. crispatus has been shown to be less abundant in the endometrium of patients with chronic endometritis (37), a condition associated with RPL (5). In contrast, L. iners, which was the most dominant microbe in the RPL women's endometrium, has been associated with dysbiosis (35) and adverse reproductive outcomes, including subfertility (38), spontaneous miscarriage (39), and preterm birth (36). Our observations support the beneficial effect of $L$. crispatus and an unfavourable effect of $L$. iners on reproductive outcomes.

We also showed an association between endometrial and vaginal $G$. vaginalis colonization and RPL. $G$. vaginalis is typically dominant in $\mathrm{BV}$, which has been associated with early $(6,7)$ and especially with late miscarriages (40). Miscarriage has also been linked with Lactobacillus depletion and high bacterial diversity in vaginal samples collected during early pregnancy (10). Kuon et al. reported that RPL women, who had vaginal G. vaginalis colonization, showed higher peripheral blood natural killer (NK) cell levels suggesting a link between dysbiotic reproductive tract microbiota, inflammation, and miscarriage (41). Furthermore, Lactobacillus depletion and the presence of pathogenic bacteria such as Bifidobacterium, Gardnerella, Klebsiella, and Neisseria in endometrial biopsies has been associated with unsuccessful reproductive outcomes in IVF treatment (42). 
Beneficial endometrial microbiota may support early pregnancy, while abnormal microbiota can be detrimental, although the underlying mechanisms are still poorly understood $(43,44,45)$. In vitro, $L$. crispatus has been shown to attach to the decidualized endometrial cells and occupy the attachment sites from pathogenetic microbes (46). Conversely, unfavourable endometrial microbiota may weaken the epithelial tight junctions, allowing pathogens to enter the endometrial stroma and induce a harmful immune reaction (43). The activation of Toll-like receptors on the surface of endometrial cells by microbial molecules may elicit the secretion of cytokines that alter the local immune environment (44), leading to poor NK cell maturation. This may provoke disturbances in placentation (43) since NK cells are essential in trophoblast invasion (47) and remodelling of spiral arteries (48). Abnormal endometrial microbiota may also favour endometrial T-helper 1 cell types (45), which is thought to predispose to RPL (49).

The origin of endometrial microbes is still unclear, but vagina and gastrointestinal tract have been suggested $(45)$. Our and others' $(29,30)$ findings of the concordance between vaginal and endometrial microbiota speak in favour of the vaginal route. Other possible mechanisms include hematogenous spreading from the gastrointestinal tract or retrograde ascension from the peritoneal cavity through fallopian tubes. Interestingly, we detected oxygen-sensitive intestinal bacteria, including Blautia and Faecalibacterium spp., in endometrial samples. Also, Verstraelen et al. (50) detected Bacteroides spp. in the endometrial samples of women with RPL or recurrent implantation failure which support the theory of peritoneal route. However, although we detected intestinal bacteria solely in the endometrial samples, it cannot be excluded that these bacteria have ascended from the perineum through vagina and remained under detection level due to unfavourable conditions, such as low pH. Finally, the role of intestinal vs. vaginal microbes in the uterus and their potential effects on reproductive health remain to be elucidated.

\section{Conclusions}

RPL was associated with an unfavourable female reproductive tract microbiota, especially in the uterus. An unhealthy endometrial microbial environment may be a new background cause for RPL, possibly contributing to an adverse immunological response during implantation and placentation. Further research should examine the mechanisms of how altered microbiota may contribute to RPL, evaluate if the prognosis of following pregnancies can be assessed according to the microbiota profile, and whether endometrial microbiota could be modified to increase the success of future pregnancies in some couples having RPL.

\section{Declarations}

\section{Acknowledgements}

We are deeply thankful for the women who participated in this study. We also wish to thank midwives Anneli Porkka, Sanna Erkkilä, and Karoliina Hyttinen for their excellent work in caring for the women and 
the schedules, laboratory coordinator Tinja Kanerva for processing the samples, and MD, MSc (Tech) Seppo Virtanen for his contribution to the study design.

\section{Disclosure of interests}

All authors declare that they have no competing interests.

\section{Contribution to authorship}

$\mathrm{PP}, \mathrm{TH}, \mathrm{IK}, \mathrm{AT}, \mathrm{AS}$, and $\mathrm{HH}$ conceived and designed the study. PP and $\mathrm{HH}$ recruited the patients, collected the samples and the clinical data. AS was responsible for the microbiota analyses. SS, PP, TH, and AS analysed the data and SS prepared the figures. PP, TH, and SS wrote the manuscript. All authors made critical reviews and approved the final version of the manuscript.

\section{Details of ethics approval}

The ethics committee of HUS approved the study (HUS/3635/2017, approval date 17 January 2018).

\section{Funding}

The European Union's Horizon 2020 research and innovation programme (H2020 MSCA Sweet Crosstalk project under grant agreement No. 814102) and the Department of Obstetrics and Gynecology, Helsinki University Hospital (No. TYH2018232) funded the study. Funds from HUS Hyvinkää Hospital (No. M3080TUT21) and The Finnish Cultural Foundation for PP, Academy of Finland for IK and The Juhani Aho Foundation for Medical Research for PP and HH.

\section{Data availability statement}

The sequencing data that support the findings of this study are available in the European Nucleotide Archive (PRJEB48310). All code has been deposited to GitHub:

https://github.com/SchahzadSaqib/TOIVE. The clinical metadata are not publicly available due to privacy but are available from the corresponding author upon reasonable request.

\section{References}

1. ESHRE Guideline Group on RPL, Bender Atik R, Christiansen OB, Elson J, Kolte AM, Lewis S, et al. ESHRE guideline: recurrent pregnancy loss. Hum Reprod Open 2018;2018:hoy004.

2. Raj R, Reagan L. Recurrent miscarriage. Lancet 2006;368:601-611.

3. McQueen DB, Perfetto CO, Hazard FK, Lathi RB. Pregnancy outcomes in women with chronic endometritis and recurrent pregnancy loss. Fertil Steril 2015;104:927-31.

4. Giakoumelou S, Wheelhouse N, Cuschieri K, Entrican G, Howie SE, Horne AW. The role of infection in miscarriage. Hum Reprod Update 2016;22:116-33. 
5. McQueen DB, Maniar KP, Hutchinson A, Confino R, Bernardi L, Pavone ME. Redefining chronic endometritis: the importance of endometrial stromal changes. Fertil Steril 2021;116:855-61.

6. Ralph SG, Rutherford AJ, Wilson JD. Influence of bacterial vaginosis on conception and miscarriage in the first trimester: cohort study. BMJ 1999;319:220-3.

7. Haahr T, Zacho J, Brauner M, Shathmigha K, Skov Jensen J, Humaidan P. Reproductive outcome of patients undergoing in vitro fertilisation treatment and diagnosed with bacterial vaginosis or abnormal vaginal microbiota: a systematic PRISMA review and meta-analysis. BJOG 2019;126:2007.

8. Maclntyre DA, Chandiramani M, Lee YS, Kindinger L, Smith A, Angelopoulos N, et al. The vaginal microbiome during pregnancy and the postpartum period in a European population. Sci Rep 2015;5:8988.

9. Freitas AC, Chaban B, Bocking A, Rocco M, Yang S, Hill JE, et al. The vaginal microbiome of pregnant women is less rich and diverse, with lower prevalence of Mollicutes, compared to non-pregnant women. Sci Rep 2017;7:9212-9.

10. Al-Memar M, Bobdiwala S, Fourie H, Mannino R, Lee YS, Smith A, et al. The association between vaginal bacterial composition and miscarriage: a nested case-control study. BJOG 2020;127:264-74.

11. Bradford LL, Ravel J. The vaginal mycobiome: A contemporary perspective on fungi in women's health and diseases. Virulence 2017;8:342-51.

12. Chen C, Song X, Wei W, Zhong H, Dai J, Lan Z, et al. The microbiota continuum along the female reproductive tract and its relation to uterine-related diseases. Nat Commun 2017;8:875

13. Koedooder R, Mackens S, Budding A, Fares D, Blockeel C, Laven J, et al. Identification and evaluation of the microbiome in the female and male reproductive tracts. Hum Reprod Update 2019;25:298325.

14. Moreno I, Codoner FM, Vilella F, Valbuena D, Martinez-Blanch JF, Jimenez-Almazan J, et al. Evidence that the endometrial microbiota has an effect on implantation success or failure. Am J Obstet Gynecol 2016;215:684-703.

15. Virtanen S, Rantsi T, Virtanen A, Kervinen K, Nieminen P, Kalliala I, Salonen A. Vaginal Microbiota Composition Correlates Between Pap Smear Microscopy and Next Generation Sequencing and Associates to Socioeconomic Status. Sci Rep 2019; 9:7750-8.

16. Virtanen S, Saqib S, Kanerva T, Nieminen P, Kalliala I, Salonen A. Metagenome-validated Parallel Amplicon Sequencing and Text Mining-based Annotations for Simultaneous Profiling of Bacteria and Fungi: Vaginal Microbiome and Mycobiota in Healthy Women. https://www.researchsquare.com/article/rs-321778/v1. 2021.

17. Saqib S. Taxonomic annotations - BLAST alignment and text-mining based filtration in R. https://github.com/SchahzadSaqib/taxminer. 2021.

18. Oksanen J, Blanchet FG, Friendly M, Kindt R, Legendre P, McGlinn D, et al. Vegan: Community Ecology Package. R package version 2.5-7. https://CRAN.R-project.org/package=vegan. 2020. 
19. Korpela K. mare: Microbiota Analysis in R Easily. R package version 1.0. https://github.com/katrikorpela/mare. 2016

20. Santiago GL, Tency I, Verstraelen $H$, Verhelst $R$, Trog $M$, Temmerman $M$, et al. Longitudinal qPCR study of the dynamics of L. crispatus, L. iners, A. vaginae, (sialidase positive) G. vaginalis, and $P$. bivia in the vagina. PLoS One 2012;7:e45281.

21. Khan KN, Fujishita A, Masumoto $H$, Muto $H$, Kitajima M, Masuzaki $H$, et al. Molecular detection of intrauterine microbial colonization in women with endometriosis. Eur J Obstet Gynecol Reprod Biol 2016;199:69-75.

22. Kadogami D, Nakaoka Y, Morimoto Y. Use of a vaginal probiotic suppository and antibiotics to influence the composition of the endometrial microbiota. Reprod Biol 2020;20:307-14.

23. Molina NM, Sola-Leyva A, Haahr T, Aghajanova L, Laudanski P, Castilla JA, et al. Analysing endometrial microbiome: methodological considerations and recommendations for good practice. Hum Reprod 2021;36:859-79.

24. Liu Y, Wong KK, Ko EY, Chen X, Huang J, Tsui SK, et al. Systematic Comparison of Bacterial Colonization of Endometrial Tissue and Fluid Samples in Recurrent Miscarriage Patients: Implications for Future Endometrial Microbiome Studies. Clin Chem 2018;64:1743-52.

25. Wei W, Zhang X, Tang H, Zeng L, Wu R. Microbiota composition and distribution along the female reproductive tract of women with endometriosis. Ann Clin Microbiol Antimicrob 2020;19:15.

26. Fang RL, Chen LX, Shu WS, Yao SZ, Wang SW, Chen YQ. Barcoded sequencing reveals diverse intrauterine microbiomes in patients suffering with endometrial polyps. Am J Trans/ Res 2016;8:1581-92.

27. Tu Y, Zheng G, Ding G, Wu Y, Xi J, Ge Y, et al. Comparative Analysis of Lower Genital Tract Microbiome Between PCOS and Healthy Women. Front Physiol 2020;11:1108.

28. O'Callaghan JL, Turner R, Dekker Nitert M, Barrett HL, Clifton V, Pelzer ES. Re-assessing microbiomes in the low-biomass reproductive niche. BJOG 2020;127:147-158.

29. Wang J, Li Z, Ma X, Du L, Jia Z, Cui X, et al. Translocation of vaginal microbiota is involved in impairment and protection of uterine health. Nat Commun 2021;12:4191-8.

30. Walther-António MR, Chen J, Multinu F, Hokenstad A, Distad TJ, Cheek EH, et al. Potential contribution of the uterine microbiome in the development of endometrial cancer. Genome Med. 2016; 8:122.

31. Winters AD, Romero R, Gervasi MT, Gomez-Lopez N, Tran MR, Garcia-Flores V, et al. Does the endometrial cavity have a molecular microbial signature? Sci Rep. 2019;9:9905.

32. Kunz G, Beil D, Deiniger H, Einspanier A, Mall G, Leyendecker G. The uterine peristaltic pump. Normal and impeded sperm transport within the female genital tract. Adv Exp Med Biol 1997;424:267-77.

33. Mitchell CM, Haick A, Nkwopara E, Garcia R, Rendi M, Agnew K, et al. Colonization of the upper genital tract by vaginal bacterial species in nonpregnant women. Am J Obstet Gynecol 2015;212:611.e1,611.e9. 
34. Miles SM, Hardy BL, Merrell DS. Investigation of the microbiota of the reproductive tract in women undergoing a total hysterectomy and bilateral salpingo-oopherectomy. Fertil Steril 2017;107:813820 .

35. Petrova MI, Reid G, Vaneechoutte M, Lebeer S. Lactobacillus iners: Friend or Foe? Trends Microbiol 2017;25:182-91.

36. Kindinger LM, Bennett PR, Lee YS, Marchesi JR, Smith A, Cacciatore S, et al. The interaction between vaginal microbiota, cervical length, and vaginal progesterone treatment for preterm birth risk. Microbiome 2017;5:6-9.

37. Liu Y, Ko EY, Wong KK, Chen X, Cheung WC, Law TS, et al. Endometrial microbiota in infertile women with and without chronic endometritis as diagnosed using a quantitative and reference range-based method. Fertil Steril 2019;112:707-17.

38. Campisciano G, lebba V, Zito G, Luppi S, Martinelli M, Fischer L, et al. Lactobacillus iners and gasseri, Prevotella bivia and HPV Belong to the Microbiological Signature Negatively Affecting Human Reproduction. Microorganisms 2020;9:10.3390/microorganisms9010039.

39. Nasioudis D, Forney LJ, Schneider GM, Gliniewicz K, France M, Boester A, et al. Influence of Pregnancy History on the Vaginal Microbiome of Pregnant Women in their First Trimester. Sci Rep 2017;7:10201.

40. Leitich $\mathrm{H}$, Kiss $\mathrm{H}$. Asymptomatic bacterial vaginosis and intermediate flora as risk factors for adverse pregnancy outcome. Best Pract Res Clin Obstet Gynaecol 2007;21:375-90.

41. Kuon RJ, Togawa R, Vomstein K, Weber M, Goeggl T, Strowitzki T, et al. Higher prevalence of colonization with Gardnerella vaginalis and gram-negative anaerobes in patients with recurrent miscarriage and elevated peripheral natural killer cells. J Reprod Immunol 2017;120:15-9.

42. Moreno I, Garcia-Grau I, Perez-Villaroya P, Gonzalez-Monfort M, Bahçeci M, Barrionuevo JM, et al. Endometrial microbiota composition is associated with reproductive outcome in infertile patients. doi: https://doi.org/10.1101/2021.02.05.21251207. 2021.

43. Al-Nasiry S, Ambrosino E, Schlaepfer M, Morre SA, Wieten L, Voncken JW, et al. The Interplay Between Reproductive Tract Microbiota and Immunological System in Human Reproduction. Front Immunol 2020;11:378.

44. Benner M, Ferwerda G, Joosten I, van der Molen, R G. How uterine microbiota might be responsible for a receptive, fertile endometrium. Hum Reprod Update 2018;24:393-415.

45. Bardos J, Fiorentino D, Longman RE, Paidas M. Immunological Role of the Maternal Uterine Microbiome in Pregnancy: Pregnancies Pathologies and Alterated Microbiota. Front Immunol 2020;10:2823.

46. Shiroda M, Manning SD. Lactobacillus strains vary in their ability to interact with human endometrial stromal cells. PLoS One 2020;15:e0238993.

47. Moffett A, Shreeve N. First do no harm: uterine natural killer (NK) cells in assisted reproduction. Hum Reprod 2015;30:1519-25. 
48. Smith SD, Dunk CE, Aplin JD, Harris LK, Jones RL. Evidence for immune cell involvement in decidual spiral arteriole remodeling in early human pregnancy. Am J Pathol 2009;174:1959-71.

49. Wang W, Sung N, Gilman-Sachs A, Kwak-Kim J. T Helper (Th) Cell Profiles in Pregnancy and Recurrent Pregnancy Losses: Th1/Th2/Th9/Th17/Th22/Tfh Cells. Front Immunol 2020;11:2025.

50. Verstraelen H, Vilchez-Vargas R, Desimpel F, Jauregui R, Vankeirsbilck N, Weyers $\mathrm{S}$, et al.

Characterisation of the human uterine microbiome in non-pregnant women through deep sequencing of the V1-2 region of the 16S rRNA gene. PeerJ 2016;4:e1602.

\section{Figures}

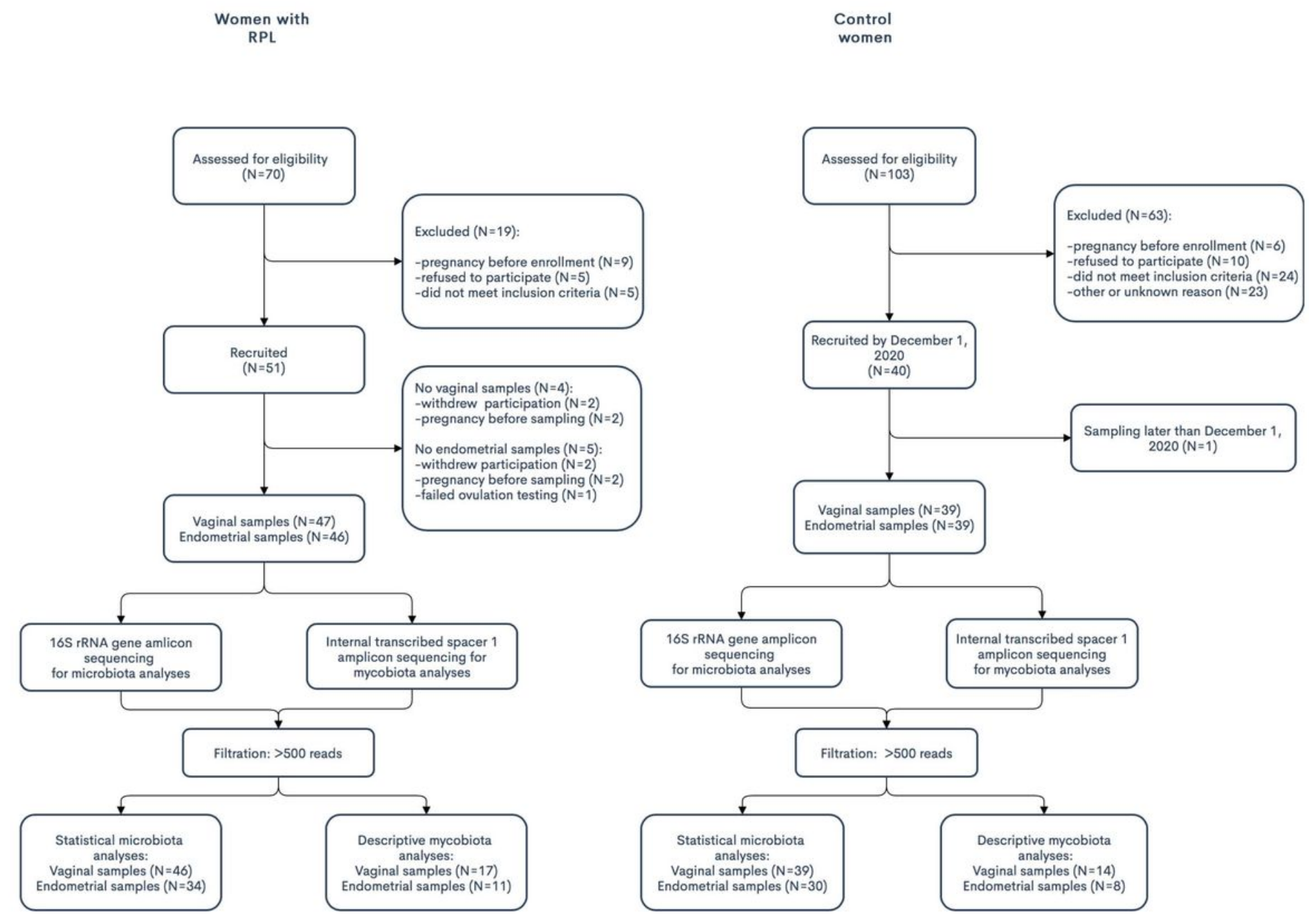

Figure 1

Flow chart describing the study design. RPL means recurrent pregnancy loss. 


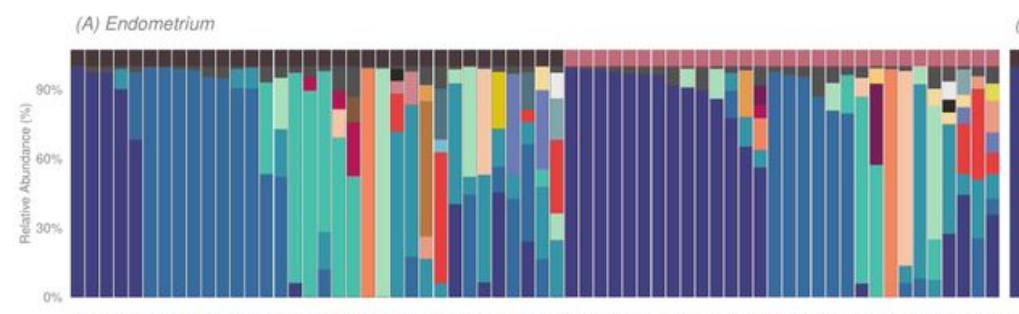

(B) Vagina
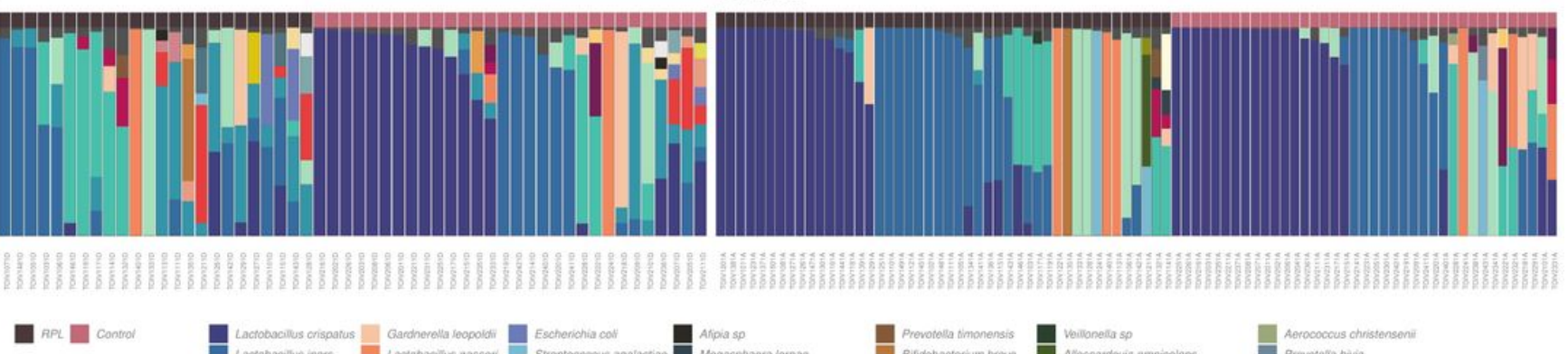

\section{Figure 2}

Stacked bar plots showing bacterial relative abundances in women with recurrent pregnancy loss (RPL) and control women, ordered based on top three most prevalent and abundant taxa (Lactobacillus crispatus, L. iners, and Gardnerella vaginalis). (A) Endometrial samples and (B) Vaginal samples. 
A)

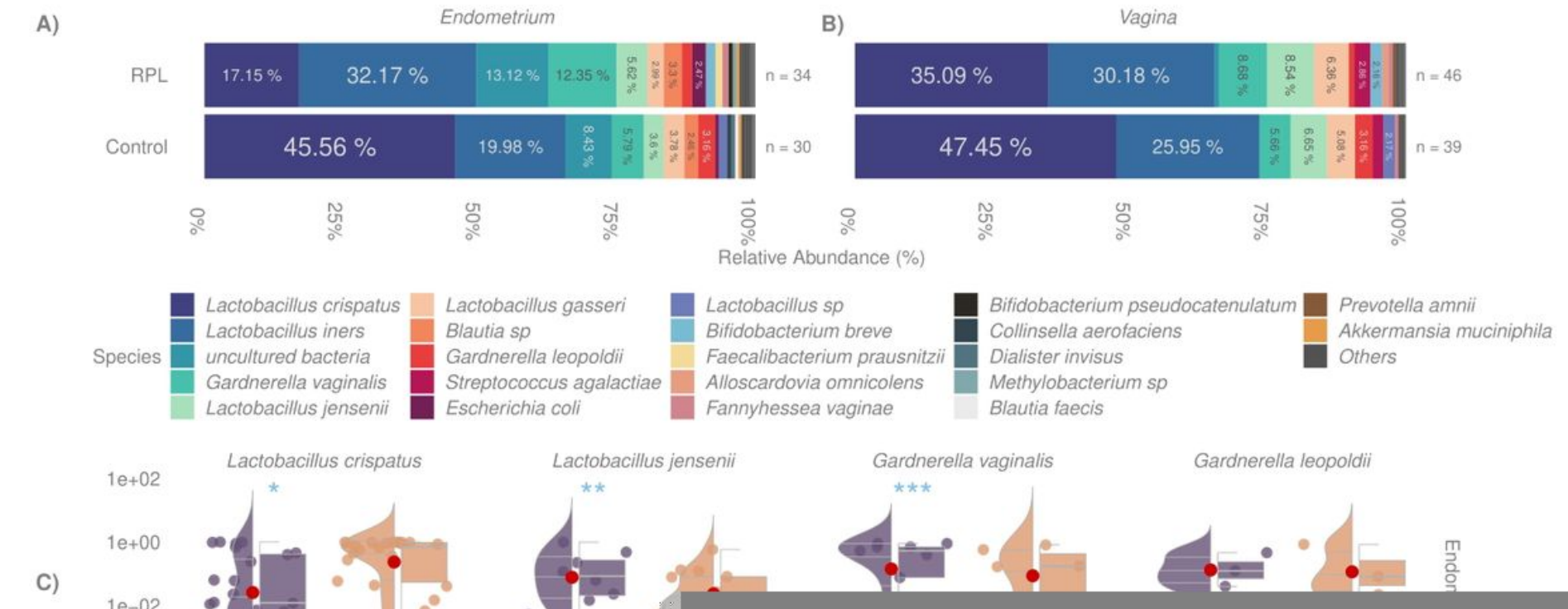

B)

Vagina

\section{Figure 3}

Illustration and summary of the main results and statistical analysis. (A-B) Bacterial mean relative abundances within the endometrial/vaginal and recurrent pregnancy loss (RPL)/control subgroups. (C E) Violin-Boxplots showing the distribution of data in each subgroup for taxa that were significantly differentially abundant after adjusting with age, parity, and BMI in the endometrial (C), vaginal (D) and pooled samples (E). Asterisks indicate whether there are any statistically significant differences between the RPL and control (reference) subgroups; ${ }^{\star \star \star} \mathrm{q}<0.001$, ${ }^{\star \star} \mathrm{q}<0.01,{ }^{*} \mathrm{q}<0.05$. Pooled means the combined results of endometrial and vaginal samples. 

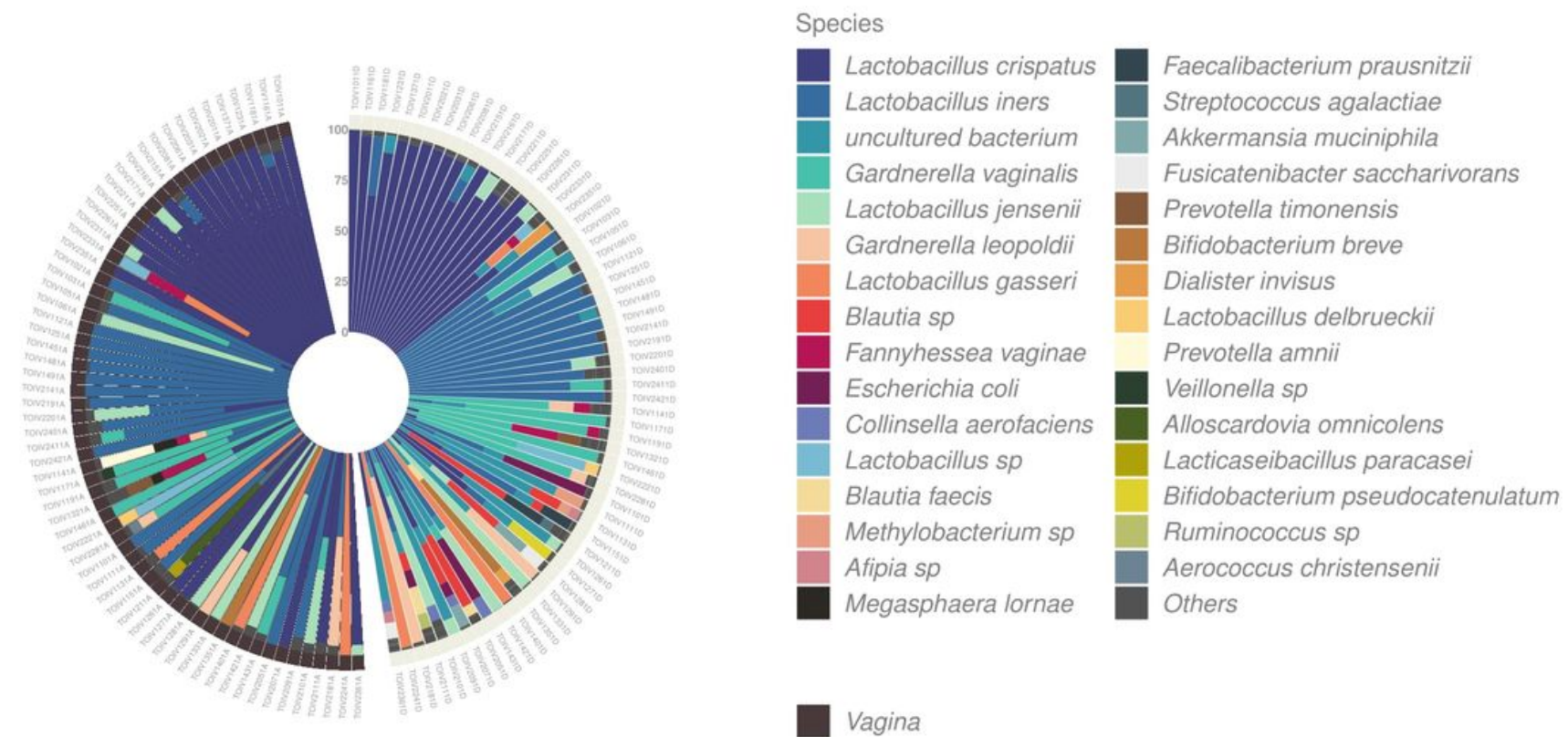

Vagina

Endometrium

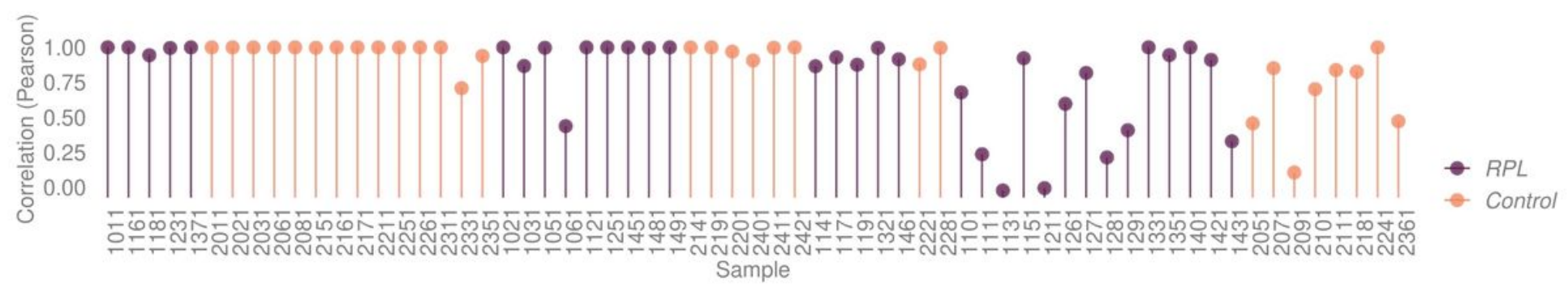

\section{Figure 4}

(A) Polar stacked bar plot illustrating the similarity of taxonomic profiles between endometrial (right) and vagina samples (left). The samples are arranged in the same order from top to bottom, creating a mirrorimage between the two semi-circles and allowing direct comparison between the sample types. (B) Pearson's correlation between the paired endometrial and vaginal samples, arranged in the same order.

\section{Supplementary Files}

This is a list of supplementary files associated with this preprint. Click to download.

- ToiveTableS1.xlsx

- ToiveTables2.xlsx

- ToiveTableS3.xIsx

- ToiveFigureS1.pdf

- ToiveFigures2.pdf

- ToiveFigures1.pdf

- ToiveFigures4.pdf 
- ToiveFigureS5.pdf

Page 19/19 